\author{
Pupil Dilation to Explicit and Non-Explicit Sexual Stimuli \\ Tuesday M. Watts and Luke Holmes \\ University of Essex \\ Ritch C. Savin-Williams \\ Cornell University \\ Gerulf Rieger \\ University of Essex
}

Author Note

Tuesday Watts and Luke Holmes, Department of Psychology, University of Essex;

Ritch C. Savin-Williams, Department of Human Development, Cornell University;

Gerulf Rieger, Department of Psychology, University of Essex.

This research was supported by the American Institute of Bisexuality, the United

States Department of Agriculture (NYC-321421), and the University of Essex.

Correspondence concerning this article should be addressed to Gerulf Rieger,

Department of Psychology, University of Essex, Colchester C04 3SQ, UK. Email:

gerulf@essex.ac.uk

Word Count: Abstract (149); Main Text (6445) 


\begin{abstract}
Pupil dilation to explicit sexual stimuli (footage of naked and aroused men or women) can elicit sex and sexual orientation differences in sexual response. If similar patterns were replicated with non-explicit sexual stimuli (footage of dressed men and women), then pupil dilation could be indicative of automatic sexual response in fully non-invasive designs. We examined in 325 men and women with varied sexual orientations, whether dilation patterns to non-explicit sexual stimuli resembled those to explicit sexual stimuli depicting the same sex or other sex. Sexual orientation differences in pupil dilation to non-explicit sexual stimuli mirrored those to explicit sexual stimuli. However, the relationship of dilation to non-explicit sexual stimuli with dilation to corresponding explicit sexual stimuli was modest, and effect magnitudes were smaller with non-explicit sexual stimuli than explicit sexual stimuli. The prediction that sexual orientation differences in pupil dilation are larger in men than in women was confirmed with explicit sexual stimuli but not with non-explicit sexual stimuli.
\end{abstract}

Keywords: pupil dilation, sexual arousal, sexual orientation, sex differences 


\section{Pupil Dilation to Explicit and Non-Explicit Sexual Stimuli}

Sexual orientation is a critical motivational drive that influences humans in forming romantic and sexual relationships, mating, and reproduction (Rahman \& Wilson, 2003). Despite this relevance, various recommendations exist regarding how to assess sexual orientation in a reliable and valid manner (Chivers, Seto, Lalumière, Laan, \& Grimbos, 2010; Korchmaros, Powell, \& Stevens, 2013; Sell, 1997). Although self-report measures are the most common, because of the stigma associated with nonheterosexual orientations, self-reports can, in some cases, be ambiguous or distorted if participants are not able or willing to fully disclose their sexual orientation (Friedman et al., 2004; Herek, 2004; Ragins, Singh, \& Cornwell, 2007). Some research has therefore focused on an automatic correlate of sexual orientation, genital arousal, which is not affected by the cognitive limitations of self-report (Janssen, Prause, \& Geer, 2007; Seto, Lalumière, \& Blanchard, 2000). It has been argued that in certain cases where self-report does not match genital response, that the latter might, in fact, be the more accurate indicator of sexual orientation (Bailey, 2009). With measures such as penile strain gauges and vaginal plethysmographs, effects of sexual orientation on genital response, and sex differences in these effects of sexual orientation, have been repeatedly described (Bailey, 2009; Huberman \& Chivers, 2015; Rieger et al., 2015a; Suschinsky \& Lalumière, 2011). Yet, a considerable disadvantage of genital arousal measurement is its invasiveness (Strassberg \& Lowe, 1995), which makes many people opt out of such experiments (Chivers, Rieger, Latty, \& Bailey, 2004; Wolchik, Spencer, \& Lisi, 1983), and those who volunteer may not represent the general population. We have therefore studied an alternative measure of automatic sexual arousal: pupil dilation to sexual 
stimuli (Rieger et al., 2015a). Participants are arguably more likely to take part in experiments that assesses their eye gaze rather than their genital response.

Pupil dilation to stimuli reflects activation of the autonomic nervous system (Lang \& Bradley, 2010), which affects other automatic and semi-automatic processes such as genital response, perspiration, digestion, blood pressure, heart rate, and breathing (ten Donkelaar, Němcová, Lammens, Overeem, \& Keyser, 2011). Pupils responding to stimuli likely indicate attention that is not in the conscious control of participants (Heaver $\&$ Hutton, 2011). For these reasons, pupil dilation has been used as a measure of automatic responses, for example, in studies on implicit reaction and cognitive load, as well as for studies on sexual interest (Goldinger \& Papesh, 2012; Laeng, Sirois, \& Gredebäck, 2012). Pupils tend to dilate more to sexual stimuli depicting an individual's preferred sex than to stimuli of the other sex or to non-sexual stimuli (Hess \& Polt, 1960; Hess, Seltzer, \& Shlien, 1965; Rieger \& Savin-Williams, 2012). In fact, dilation to sexually preferred stimuli appears to be the strongest pupillary response elicited by stimuli (Laeng et al., 2012). These findings point to the utility of this measure for research on sexual attraction and arousal.

In general, pupil dilation patterns to sexual stimuli mirror genital arousal to such stimuli: with either measure, the link of sexual orientation with sexual responses to the same sex or other sex is stronger in men than in women (Bailey, 2009; Chivers, Seto, \& Blanchard, 2007; Rieger et al., 2015a; Rieger \& Savin-Williams, 2012). This difference is most obvious when sexual orientation is related to a contrast score of sexual response (i.e., responses to the same sex minus responses to the other sex). In men, such relationships tend to be strong in effect. Heterosexual men respond most to the other sex, homosexual 
men respond most to the same sex, and bisexual men show, on a group level, responses that are in-between those of heterosexual and homosexual men (Bailey, Rieger, \& Rosenthal, 2011; Rieger et al., 2013; Savin-Williams, Rieger, \& Rosenthal, 2013). In women, both pupil dilation and genital arousal patterns are weakly related to their sexual orientation, largely because heterosexual and bisexual women respond similarly to both sexes, whereas lesbians are somewhat more aroused to their preferred sex (women) than the other sex (Chivers et al., 2007; Rieger, Savin-Williams, Chivers, \& Bailey, 2015b). In addition to similar sex differences in the relationship of sexual orientation with sexual response, pupil dilation and genital arousal patterns show a similarity in a further sex difference. The correspondence of these measures with each other and with other responses to sexual stimuli, including subjective arousal, or time spent viewing these stimuli, is stronger in men than in women (Chivers et al., 2004; Chivers et al., 2010; Rieger et al., 2015a; Rieger \& Savin-Williams, 2012). It is possible that for men, more than for women, stronger congruencies between various indices of their sexual arousal aid them in staying sexually oriented towards relevant targets (Rieger et al., 2015a). But regardless of the ultimate reasons for these differences, distinctions between the sexes and between sexual orientation groups, as reported with genital arousal measures, can be replicated with the assessment of pupillary response.

In the research reviewed thus far, highly explicit sexual stimuli have been employed (for example, two people engaged in sexual intercourse or one person masturbating). For the assessment of genital arousal, highly explicit sexual stimuli elicit the strongest responses (McConaghy, 1999), and for similar reason, explicit sexual stimuli have also been used for assessing pupil dilation (Hess \& Polt, 1960; Rieger et al., 
2015a; Savin-Williams et al., 2013). However, viewing such stimuli can be invasive in itself and may limit researchers in recruiting a diverse range of participants. Moreover, in many countries the viewing of explicit sexual stimuli is illegal, restricting research with such measures to Westernized societies that may not be representative of the global population (Henrich, Heine, \& Norenzayan, 2010). Because non-explicit sexual stimuli, such as footage of attractive but dressed men and women, are for some participants likely to be less invasive than explicit sexual stimuli, using such non-explicit sexual stimuli can be helpful for research on populations where it is inadvisable or unethical because of the participant's age, personal values, or cultural context to show explicit sexual stimuli.

As pupils are highly sensitive to attention-triggering stimuli, and this even within a few seconds of exposure to stimuli (Bradley, Miccoli, Escrig, \& Lang, 2008; Laeng et al., 2012), they might, in fact, not need the same degree of explicit sexual stimulation as genitals do to show reliable reactions that correspond with a person's sexual orientation or sex. Thus, if pupil dilation to non-explicit sexual stimuli relates to dilation to explicit sexual stimuli, then dilation patterns with the first measure could reflect dilation patterns with the second measure (and, by extension, reflect previously described genital responses to explicit sexual stimuli). Support for the possibility that non-explicit sexual stimuli elicit responses similar to explicit sexual stimuli comes from another measure of gaze, viewing time. Time spent viewing stimuli depicting swimsuit models yielded attraction patterns that mirror patterns of genital arousal: on average, men viewed their preferred sex more strongly than women did, but within women, homosexual women tended to view their preferred sex more strongly than heterosexual women did (Lippa, 2012). Similar sex and sexual orientation differences were described in another study 
with a viewing time paradigm (Ebsworth \& Lalumière, 2012). Moreover, a recent study suggests that heterosexual men dilated to female models but not to male models in swim suits, whereas heterosexual women dilated to both sexes (Attard-Johnson, Bindemann, \& Ó Ciardha, 2016). In combination, these studies raise the possibility that predicable sex and sexual orientation differences are elicited with pupil dilation to non-explicit sexual stimuli.

It is further possible that any responses elicited with non-explicit sexual stimuli, such as dressed men and women, will not be as strong as responses elicited with explicit sexual stimuli. Sex differences in viewing time and gaze fixation are stronger with explicit sexual stimuli than with non-explicit sexual stimuli, although sex differences can still be elicited with non-explicit sexual stimuli (Aboyoun \& Dabbs, 1998; Ebsworth \& Lalumière, 2012; Lykins, Meana, \& Kambe, 2006; Lykins, Meana, \& Strauss, 2008). Similarly, sex and sexual orientation differences in pupil dilation may be stronger with explicit than non-explicit sexual stimuli.

Based on the reviewed literature, the following predictions were tested:

\section{Prediction 1}

Pupil dilation to explicit sexual stimuli depicting males or females will relate to pupil dilation to corresponding non-explicit sexual stimuli.

\section{Prediction 2}

For both explicit and non-explicit sexual stimuli, the correspondence of pupil dilation to male or female sexual stimuli with self-reported sexual orientation will be stronger in men than women.

\section{Prediction 3}


For both explicit and non-explicit sexual stimuli, the concordance of pupil dilation to sexual stimuli with time spent viewing these stimuli and self-reported sexual attraction to these stimuli will be stronger in men than women.

\section{Prediction 4}

For both explicit and non-explicit sexual stimuli, the relationships of pupil dilation with self-reported sexual orientation, viewing time of stimuli, and self-reported sexual attraction to these stimuli will be significant, although stronger for explicit than nonexplicit sexual stimuli.

\section{Method}

\section{Participants}

The recruitment period was from October 2010 to June 2011. Advertisements were placed on several websites at a Northeast university in the USA. In addition, advertisements for bisexual men were placed at local web forums where men sought sexual encounters with either men, women, or both. The method is efficient for recruiting bisexual-identified men, who are less common than other men (Rosenthal, Sylva, Safron, \& Bailey, 2012). The 165 recruited men self-identified as "exclusively straight" $(n=31)$, “mostly straight” $(n=24)$, "bisexual leaning straight” $(n=15)$, "bisexual” $(n=10)$, "bisexual leaning gay" $(n=21)$, "mostly gay" $(n=33)$, and "exclusively gay" $(n=31)$.

The 160 recruited women self-identified as "exclusively straight" $(n=34)$, "mostly straight" $(n=27)$, "bisexual leaning straight" $(n=11)$, "bisexual" $(n=17)$, "bisexual leaning lesbian" $(n=16)$, "mostly lesbian" $(n=32)$, and "exclusively lesbian" $(n=23)$. The average age $(S D)$ was $23.36(6.62)$ years in men and $27.70(6.78)$ years in women. The most common ethnicity was Caucasian in men (64\%) and women (69\%). Mixed 
ethnicities were second most common across men and women (10\%), followed by Asian, African-American, Hispanic, and Native-American. For these participants, results for pupil dilation patterns to explicit sexual stimuli have been previously published (Rieger \& Savin-Williams, 2012), but findings for non-explicit sexual stimuli and a comparison of pupil dilation patterns to the two types of stimuli have not been reported.

\section{Measures}

Sexual orientation. Participants reported their aforementioned sexual orientation identities, in addition to their sexual attractions, sexual fantasies, and sexual infatuations (i.e., passions for men or women) on Kinsey-type scales (Kinsey, Pomeroy, \& Martin, 1948). For example, for sexual attraction, participants endorsed one of seven statements ranging from " $0=$ exclusively attracted to the females", to " $3=$ equal attraction to females and males," to " $6=$ exclusively attracted to males." The four measures were correlated in men (all $p$ 's $<.0001$, all $r$ 's $\geq .95, .92<$ CI's $>.99$ ) and women (all $p$ 's $<$. 0001, all $r$ 's $\geq .92, .89<$ CI's $>.96$ ), and averaged within participants. An average score of 0 represented an exclusively heterosexual orientation and an average score of 6 an exclusively homosexual orientation.

Stimuli. Each stimulus was a 30 -second video of similar luminance. Twelve sexually explicit male stimuli and 12 sexually explicit female stimuli depicted a full body shot of either a naked male or female model, alone in a room, sexually aroused, and masturbating. These stimuli were selected from a pool of 200 videos drawn from sites on the Internet. In a pilot study, heterosexual and homosexual men and women rated these videos on the models' sexual appeal, and those stimuli that were rated highest across all groups were used for this study. 
The 12 non-explicit male and 12 non-explicit female stimuli depicted men and women who were casually dressed, in full view, and seated in a chair discussing winters in North America. Their answers were given to the interviewer who was standing behind the camera. These men and women were originally interviewed at a Midwestern university in the USA. In total there were 185 non-explicit sexual stimuli whose attractiveness has been previously assessed (Rieger et al., 2011), and the most attractive men and women were used in the present study. The average age $(S D)$ was $21.67(1.72)$ years and 22.83 (3.83) years, respectively. The most common ethnicity was Caucasian with $67 \%$ and $58 \%$, respectively.

Two 1-minute videos of landscapes, taken from nature documentaries, were used as neutral stimuli.

Pupil data. An SR Research Remote infrared gaze tracker recorded participants' eyes. The gaze tracker collected data every two milliseconds with a $16 \mathrm{~mm}$ lens focused on participants' preferred eye. Participants' heads rested on a mount $500 \mathrm{~mm}$ from the lens, and the head's exact position was automatically recorded by measuring the distance in $\mathrm{mm}$ from the lens to a reference point affixed to the forehead. The program EyeLink computed pupil area as the number of the tracker's camera pixels occluded by the infrared light reflected by the pupil. If pupils dilated while viewing stimuli, more pixels were occluded.

Viewing time. Viewing time was captured with the SR Research gaze tracker, and calculated as the percentage of time looking at a male or female sexual stimulus.

\section{Procedure}


After giving written informed consent, participants took seat in a dimly lit room facing the gaze tracker underneath a monitor with a screen resolution of 1024 by 768 pixels. Stimuli were presented in two modules, the first of which was used for recording pupil dilation data. Participants watched a neutral stimulus, followed, in random order, by the sexual stimuli. Participants randomly watched either all the explicit sexual stimuli first or all the non-explicit sexual stimuli first. After each stimulus, participants answered three questions in random order, regarding how sexually attractive they found the person, how sexually appealing they found the person, and how much they would like to date the person. These questions were displayed across the screen and participants used a mouse to give their answers. Participants responded to each question with a 7-point scale ranging from "not at all" to "average," to "very much." Immediately after they answered the last question, the next stimulus was presented.

The second module started with another neutral stimulus. Then, two stimuli were presented simultaneously. Half of these paired stimuli showed the male to the right of the female; the other half showed the male to the left of the female. Participants were free to choose how much time they wanted to spend on viewing either of the paired stimuli. Participants watched either all paired explicit sexual stimuli first, or all paired nonexplicit sexual stimuli first. For each type of stimulus, paired stimuli were shown in random order. This module was chosen for the collection of data regarding time spent viewing male or female stimuli. After each stimulus pair, participants answered three questions that were, in random order, displayed across the screen. They responded to which of the two people they found more sexually attractive, more sexually appealing, and would more like to date. Answers were given on 7-point scales ranging from "very 
much the left," to "equal," to "very much the right." Because a male stimulus could be either to the left or right of a female stimulus, we considered it would be easier for participants to indicate on which side the more attractive model was, rather than stating that they were more attracted to the "male" or "female."

For each module, participants eye movements were continuously monitored in a control room with a screen that showed the stimuli participants were viewing, with the position of their pupil superimposed on it. In the rare cases that participants looked away from the screen, they were instructed through an intercom that they should watch every video carefully, no matter if they liked the content or not. Other than that participants were free to view any part of the screen as they wished.

After the experiment, participants completed a survey with demographic information and sexual orientation and received payment. The procedure took approximately 45 minutes.

\section{Data Reduction}

For each participant and for each stimulus, pupil size data were averaged by dividing the total of occluded camera pixels across data points by the number of data points (which were collected every two milliseconds). Similarly, for each participant and stimulus, we calculated average head distance by dividing the total of head distance (in $\mathrm{mm}$ ) across data points by the number of data points. Averaged pupil size was multiplied with averaged head distance for each stimulus, to account for some head movements between stimuli. For further standardization of data, we followed procedures that yield predicted sex and sexual orientation differences in pupil dilation data (Rieger et al., 2015a), and that mirror recommendations for analyzing genital arousal responses (Harris, 
Rice, Quinsey, Chaplin, \& Earls, 1992). Because pupils vary in size and level of dilation, we computed within each participant z-scores of pupillary response to each stimulus. We then calculated, for each participant, five mean values, one each for average pupil dilation to explicit male sexual stimuli, explicit female sexual stimuli, non-explicit male sexual stimuli, non-explicit female sexual stimuli, and neutral stimuli. For analyses of pupil dilation to explicit and non-explicit sexual stimuli, responses to neutral stimuli were subtracted. Hence, these variables indicated changes of pupil dilation to sexual stimuli as compared to neutral.

For viewing time, a score of $50 \%$ indicated equal time spent viewing the same sex and other sex, larger values indicated higher percentage of time viewing the same sex than the other sex, and lower values indicated the opposite. These percentages were reliable across paired explicit sexual stimuli and across paired non-explicit sexual stimuli (Cronbach's $\alpha \geq .92$ in both sexes). They were averaged within participants and separately for each stimulus type (i.e., explicit and non-explicit sexual stimuli).

For both explicit and non-explicit sexual stimuli, participants' three ratings were reliable within each stimulus, across all male stimuli, across all female stimuli, and across all paired male-female stimuli, (all $r$ 's $\geq .88$; all Cronbach's $\alpha$ 's $\geq .93$ ). For each participant and each stimulus type, an average was computed across ratings. For both explicit and non-explicit sexual stimuli collected during the first module, these averages represented self-reported sexual attraction to stimuli of the same sex, sexual attraction to stimuli of the other sex, and, from the second module, a contrast of sexual attraction to the same sex over the other sex.

\section{Results}




\section{Prediction 1}

The first prediction states that pupil dilation to explicit sexual stimuli relates to pupil dilation to corresponding non-explicit sexual stimuli. In men, there were small to modest correlations between pupil dilation to explicit and matching non-explicit sexual stimuli. This was the case for the correlation between responses to explicit and nonexplicit sexual stimuli of the same sex, $p=.03, r=.18,95 \%$ CI $[.02, .32]$, the correlation between responses to explicit and non-explicit sexual stimuli of the other sex, $p<.0001, r$ $=.40,95 \% \mathrm{CI}[.26, .52]$, and the correlation of a contrast for explicit sexual stimuli (responses to the same sex minus the other sex) with such contrast for non-explicit sexual stimuli, $p<.0001, r=.31,95 \%$ CI $[.17, .44]$. (From here on we omit the phrase "95\% CI." Instead, we simply report numbers is squared brackets to the right of an effect size in order to represent its $95 \%$ confidence intervals). In women, these correlations were similar in magnitude, $p<.0001, r=.35[.21, .48], p=.02, r=.16[.01, .31]$, and $p=.001$, $r=.25[.11, .39]$, respectively. Hence, dilation to sexual stimuli somewhat resembled dilation to non-sexual stimuli. These correlations pointed to the possibility that each of these measures - dilation to explicit and non-explicit sexual stimuli - had similar relations with sexual orientation.

\section{Prediction 2}

The second prediction states that the correspondence of pupil dilation to same-sex or other-sex stimuli with self-reported sexual orientation is stronger in men than women. We tested this prediction separately for explicit and non-explicit sexual stimuli.

Explicit Sexual Stimuli. In both men and women, pupil dilation to explicit sexual stimuli of the same sex correlated strongly and negatively with dilation to explicit sexual 
stimuli of the other sex, $p<.0001, r=-.82$ [-.87, -.76], and $p<.0001, r=-.77[-.82,-.69]$, respectively. We therefore computed a contrast score. Positive numbers resembled more dilation to the same sex than other sex, negative numbers resembled more dilation to the other sex than same sex, and zero indicating equal dilation to both sexes. We regressed this contrast onto self-reported sexual orientation. Independent variables included both the linear and quadratic function of sexual orientation because these relations can be, to some degree, curvilinear (Rieger et al., 2013; Rieger et al., 2015b).

Figure 1A shows that for men, the linear relationship (but not the curvilinear relationship) of pupil dilation to the same sex or other sex with sexual orientation was significant, $p<.0001, \beta=.54[.40, .67]$. (The effect size $\beta$ is the standardized regression coefficient that can be interpreted similarly to a correlation coefficient.) Heterosexual men dilated most to the other sex, bisexual men dilated approximately equally to both sexes, and homosexual men dilated most to the same sex.

In women, pupil dilation to the same or other sex also related to their sexual orientation in a linear fashion, $p<.0001, \beta=.44[.31, .59]$. This linear relationship was qualified by quadratic effect, although not significantly so, $p=.08, \beta=.13[-.08, .34]$. In Figure 1B, this quadratic effect can be explained by the position of the coefficient's $95 \%$ confidence intervals relative to a dilation contrast score of zero (which stands for equal dilation). Exclusively heterosexual women dilated more to the other sex than to the same sex; however, in comparison, exclusively homosexual women dilated more strongly to the same sex than the other sex. 
A multiple regression analysis tested for sex differences in the above findings. Pupil dilation to the same sex or other sex was predicted by the linear and curvilinear effects of sexual orientation, by participants' sex, and the interactions of the linear and curvilinear effects of sexual orientation with sex. These interactions examined whether the sexes differed in these linear and curvilinear effects. Results indicated no significant sex difference for the linear relation of pupil dilation with sexual orientation, $p=.35, \beta=-.04$ $[-.14, .06]$. In magnitude, the sex difference for the curvilinear relationship was stronger than for the linear relationship, but it, too, was not significant, $p=.08, \beta=.16[-.01, .28]$. If anything, the curvilinear relationship of pupil dilation patterns with sexual orientation was more common in women than men (Figure 1).

Figure 1 illustrates a further sex difference for exclusively heterosexual men and women (Kinsey Score of 0). These heterosexual men dilated more strongly to the other sex than the same sex, compared with heterosexual women. An independent-sample t-test, comparing these men and women for dilation to the other sex over the same sex, suggested a significant sex difference in effect, $p=.0001$, Cohen's $d=1.07[.81,1.31]$.

Non-Explicit Sexual Stimuli. We repeated aforementioned analyses with nonexplicit sexual stimuli. For men, pupil dilation to the same sex correlated significantly and negatively with dilation to the other sex, $p=.009, r=-.20[-.35,-.05]$. For women, this correlation was similar in magnitude, but it was not significant, $p=.09, r=-.14[-$ $.28, .02]$. A multiple regression analysis indicated that across men and women this relationship was significant and negative, $p=.002, \beta=-.17[-.29,-.06]$, and that men and women did not significantly differ in effect, $p=.54, \beta=-.03[-.15, .30]$. 
These effects were much weaker than in the previous analyses on explicit sexual stimuli. For comparison with the contrast score calculated for explicit sexual stimuli (Figure 1), we computed a corresponding contrast for non-explicit sexual stimuli by subtracting dilation to the other sex from dilation to the same sex. This contrast correlated slightly better with self-reported sexual orientation than either of its components (compare Figure 2 with Table 2). Moreover, this relation of pupil dilation to the same sex or other sex with sexual orientation was linear and significant in both men and women; $p$ $<.0001, \beta=.46[.32, .61]$, and $p<.0001, \beta=.41[27.56]$, respectively. Heterosexual men and women dilated somewhat more to the other sex than the same sex, bisexual participants showed equal dilation, and homosexual men and women dilated more to the same sex than other sex. The curvilinear effects of sexual orientation on pupil dilation were not significant (Figure 2).

Figure 2

A multiple regression analysis did not confirm that the sexes differed significantly in the above effects, neither for the linear relation of pupil dilation with sexual orientation, $p=.63, \beta=-.03[-.13, .08]$, nor for the curvilinear relationship of pupil dilation with sexual orientation, $p=.88, \beta=.01[-.15, .17]$. Furthermore, exclusively heterosexual men did not show significantly more pupil dilation to the other sex than the same sex, compared with exclusively heterosexual women, $p=.25$, Cohen's $d=.31[.06, .55]$. Thus, the correspondence of dilation patterns to non-explicit sexual stimuli with sexual orientation, as illustrated in Figure 2, was similar across men and women.

\section{Prediction 3}


We predicted that for both explicit and non-explicit sexual stimuli, pupil dilation would relate to time spent viewing stimuli and self-reported sexual attraction to stimuli. Furthermore, these correspondences would be stronger in men than women.

Explicit sexual stimuli. Table 1 shows the correlations across pupil dilation to the same sex and other sex, viewing time of same-sex or other-sex stimuli, self-reported sexual attraction towards stimuli, and of self-reported sexual orientation. Overall, these correlations were modest to strong in effect, suggesting that pupil dilation is a reliable indicator of sexual attraction, viewing time, and sexual orientation. All correlations were significant, with the exception of the correlation between self-reported sexual attraction to same-sex stimuli and other sex-stimuli for women.

\section{Table 1}

The average absolute correlation was .64 and .53 in men and women, respectively. We then computed the difference between men and women for each correlation (within pairs of variables) and tested the average of these difference scores against 0 by conducting a repeated-measures t-test. The sex difference was significant, $p<.0001$, Cohen's $d=1.96[1.66,2.27]$. (In the case of these repeated-measures t-tests, $d$ was calculated by dividing the average of correlation difference scores by its standard deviation). In a second step, we excluded all correlations with sexual orientation (Table 1) from these calculations, because sexual orientation was, unlike the other variables, not a measure of immediate responses to stimuli. The average absolute correlations remained similar to the above, with .60 in men and .49 in women. This sex difference was also significant, $p<.0001$, Cohen's $d=1.78[1.42,2.14]$. 
Non-Explicit sexual stimuli. Table 2 shows correlations as discussed in the last section, but in this case for non-explicit sexual stimuli. In contrast to correlations for explicit sexual stimuli (Table 1) correlations for non-explicit sexual stimuli varied more strongly in magnitude. Still, the majority of these correlations were significant. Exceptions were the correlation between self-reported sexual attraction to same-sex stimuli and other sex-stimuli for men, and the three correlations between pupil dilation to the other sex, self-reported sexual attraction to the same sex, and self-reported sexual attraction to the other sex for women.

Table 2

For non-explicit sexual stimuli, the average absolute correlation was .47 in men and .41 in women. This difference was weaker than in analyses for explicit sexual stimuli, but still significant, $p=.009$, Cohen's $d=0.62[0.25,0.96]$. Once correlations with sexual orientation (Table 2) were excluded from these calculations, then the respective average absolute correlations were .41 and .34 ; this difference was still significant, $p$ $=.03$, Cohen's $d=0.68[0.32,1.04]$.

\section{Prediction 4}

We predicted that the relationships of pupil dilation patterns with sexual orientation, viewing time, and self-reported attraction to stimuli would be significant with both explicit and non-explicit sexual stimuli, but that these effects would be stronger for explicit sexual stimuli than non-explicit sexual stimuli. For the relationship of pupil dilation with sexual orientation, we compared effects for explicit and non-explicit sexual stimuli as depicted in Figures 1 and 2. For the relationship of pupil dilation with viewing 
time and self-reported attraction to stimuli, we compared effects for explicit and nonexplicit sexual stimuli as shown in Tables 1 and 2.

Figures 1 and 2 illustrate that the linear relationship of pupil dilation to the same sex or other sex with self-reported sexual orientation was somewhat smaller for non-explicit sexual stimuli than explicit sexual stimuli. Repeated measures regression analyses indicated that this difference in effect was significant in men, $p=.046, \beta=.16[.00, .31]$, and not significant in women, $p=.14, \beta=.12[-.04, .27]$. We then conducted a multiple regression analysis. The dependent variable was the difference between explicit and nonexplicit sexual stimuli with respect to dilation to the same sex or other sex. Independent variables were the participants' sexual orientation, sex, and their interaction. The nonsignificant interaction suggested that there was no reliable sex difference in effect, $p=.72$, $\beta=-.02[-.13, .09]$, and across all men and women, pupil dilation to the same sex over other sex was more strongly related to sexual orientation with explicit sexual stimuli than non-explicit sexual stimuli, $p=.01, \beta=.14[.03, .25]$.

Figures 1 and 2 further illustrate that the most diminished response to non-explicit sexual stimuli (compared with explicit sexual stimuli) was for exclusively heterosexual men. We tested with an additional regression analysis whether the diminished effect was more substantial for exclusively heterosexual men than for other participants. Although this difference in effect was not significant, $p=.27, \beta=.14[-.06, .27]$, it may still be meaningful, as we will discuss below.

Tables 1 and 2 show that, in general, correlations of pupil dilation with all viewing time, self-reported sexual attraction, and self-reported sexual orientation were stronger in magnitude with explicit sexual stimuli than with non-explicit sexual stimuli. 
For both men and women, we computed the difference in absolute correlations between explicit and non-explicit sexual stimuli (within pairs of variables) and tested these difference scores against 0 with a repeated-measures t-test. In men, these correlations with explicit sexual stimuli were significantly stronger than correlations with non-explicit sexual stimuli, $p<.0001$, Cohen's $d=1.52$ [1.22, 1.82]. A similar difference was found in women, $p=.001$, Cohen's $d=0.79[0.48,1.09]$. When correlations with sexual orientation were excluded, the average absolute correlation was also stronger with explicit sexual stimuli than non-explicit sexual stimuli, both for men, $p<.0001$, Cohen's $d=1.42[1.06,1.78]$, and for women, $p=.004$, Cohen's $d=0.92[0.56,1.23]$.

\section{Discussion}

Findings of the present study suggest that a) pupil dilation to explicit and nonexplicit sexual stimuli was related, although this relationship was modest at best, b) sexual orientation differences were found with both types of stimuli, however, some sex differences were only detected with explicit but not with non-explicit sexual stimuli, and c) pupil dilation patterns were weaker with non-explicit sexual stimuli than explicit sexual stimuli.

\section{Explicit and Non-Explicit Sexual Stimuli}

Prediction 1 was confirmed in the sense that significant links in responses to explicit sexual stimuli and corresponding non-explicit sexual stimuli were found in the data. Yet, these links were weak to modest in magnitude, suggesting that pupil dilation to non-explicit sexual stimuli can only to a limited degree be interpreted as a reflection of pupil dilation to explicit sexual stimuli. This suggests that either type of sexual stimulus (explicit or non-explicit) can relate to any other variable (such as sex or sexual 
orientation) in somewhat different manners. This discrepancy could, in part, explain why sex differences in the relation of sexual orientation with pupil dilation to explicit sexual stimuli were not reflected in corresponding sex differences with non-explicit sexual stimuli.

To be certain, Predictions 2 and 3, regarding sex differences in the correspondences of pupil dilation with sexual orientation, viewing time, and self-reported attraction to stimuli were broadly confirmed: overall, these effects were stronger for men than for women (Figures $1 \& 2$, Tables \& 2). The one exception was that with non-explicit sexual stimuli, the link between sexual orientation and pupil dilation to the same sex or other sex was not significantly stronger in men than women. This was contrarily to Prediction 2. Notably, Figures 1 and 2 indicate that the most visual decrease in dilation from explicit and non-explicit sexual stimuli was for exclusively heterosexual men. This decrease made their dilation patterns to non-explicit sexual stimuli similar, in magnitude, to the dilation patterns of heterosexual women. Although this change was not significantly stronger in heterosexual men than other participants, it may have some meaning. Heterosexual men might be particularly focused on signals of female proceptivity (Mishra, Clark, \& Daly, 2007), which are likely more readily observed in nude women than dressed women. The lack of such information in non-explicit sexual stimuli could have led to a decrease in heterosexual men's arousal patterns, resulting in an overall nonsignificant sex difference in the effect of sexual orientation on pupil dilation with nonexplicit sexual stimuli.

Prediction 4 was that the correspondences of pupil dilation with sexual orientation, viewing time, and self-reported attraction would be weaker with non-explicit sexual 
stimuli than with explicit sexual stimuli. Such differences in effect were generally confirmed. With respect to sexual orientation, though, Figures 1 and 2 illustrate that these effects were not drastically weaker for non-explicit sexual stimuli than explicit sexual stimuli. Hence, the more important conclusion may be that for both men and women, it was still possible to provoke pupillary responses with non-explicit sexual stimuli that were indicative of their sexual orientation.

It is possible that any predicted differences in pupil dilation between and within the sexes will become larger in effect with refined methodologies. For example, at least for men and with explicit sexual stimuli, longer videos (3 minutes) elicit much stronger links between their pupil dilation patterns and sexual orientation than the 30 s videos used in the present research (Rieger et al., 2015a). Non-explicit videos of 3 minutes could also yield stronger effects than presently reported, and possibly reveal differences in responses to non-explicit sexual stimuli that were not detected in the current study.

Further research should examine the within-participant relationship between pupil dilation to non-explicit sexual stimuli with genital responses to explicit sexual stimuli. Such a method will aid in directly testing the prediction that pupillary responses to subtle sexual stimuli can be a reflection of genital arousal to explicit sexual stimuli. Furthermore, in qualitative interviews some of our participants stated that they felt more drawn to the non-explicit sexual stimuli than the explicit sexual stimuli, because unlike the latter, the first represented realistic romantic and sexual partners they could potentially meet (SavinWilliams, 2016). Examining features other than physical attractiveness that make nonexplicit sexual stimuli sexually or romantically appealing could enhance any relationships of the perceivers' sexual orientation with their pupil responses. 


\section{Limitations}

The presentation of stimuli was random for each set (explicit or non-explicit sexual stimuli). Hence, at the onset of stimulus presentation, each stimulus had the same chance to appear in any given order. Still, by chance, certain stimuli might have been presented earlier than others, on average, which could have affected results. However, we consider this possibility very unlikely. For each participant, the chance that a given stimulus was first in order was $1 / 12=.08$. For the same stimulus to always be first in order across all 325 participants was .08 to the power of 325 , which approximates zero. The chance that any two or more stimuli were always in the same order across all participants is even less.

Similarly, the fact that some participants viewed explicit sexual stimuli before nonexplicit sexual stimuli, or vice versa, could have affected their responses. However, the random presentation with the large number of participants should balance out any order effects.

Another limitation was that each stimulus was presented immediately after the questions for the previous stimulus; thus, undesired carry-over effects such as cognitive load could have affected pupillary response to subsequent stimuli. For this reason, we have previously reported analyses for explicit sexual stimuli that were restricted to the last 10 seconds of each stimulus. Any correlations with other variables (such as sexual orientation) were virtually identical, regardless of whether the full length of stimuli or their last 10 seconds were used for analyses (Rieger \& Savin-Williams, 2012). Likewise, results were virtually identical, regardless of whether pupil data for non-explicit sexual stimuli were restricted to the last 10 seconds or not. Hence, it is unlikely that carry-over effects influenced results. Still, a refined methodology could avoid these limitations, for 
example by presenting neutral stimuli between sexual stimuli, therefore facilitating a return to baseline before the next sexual stimulus (Rieger et al., 2015a).

\section{Conclusion}

These potential methodological improvements aside, the present data support the proposal that the assessment of pupil dilation can be combined with non-explicit sexual stimuli for fully non-invasive studies on sexual attraction and arousal (Rieger \& SavinWilliams, 2012). Such a design has advantages. For example, in several traditional cultures it is impossible to assess physiological genital arousal or to show footage that is highly graphic and sexual in content. For this reason, little is understood about the physiological sexual arousal patterns outside of Westernized societies. For instance, globally and through history, there have been men and women who do not adopt stereotypical male or female identities (Mirandé, 2015; Nanda, 1986; Young, 2000). The degree to which their identities reflect unique sexual arousal patterns is largely unknown. One such group is found in the Polynesian population of Samoa. Recent research in this population on men with varied sexual identities has used viewing time paradigms in order to examine cultural differences in attention and attraction to non-explicit sexual stimuli (Petterson, Dixson, Little, \& Vasey, 2015, 2016). Because viewing time can be to some level under the conscious control of participants (Johnson, 1995; Munoz \& Everling, 2004), using the additional assessment of pupil dilation to stimuli in such societies could give further insight into the automatic expression of universally similar or culturally distinct sexual attraction and arousal patterns. 


\section{References}

Aboyoun, D. C., \& Dabbs, J. N. (1998). The Hess pupil dilation findings: Sex or novelty? Social Behavior and Personality, 26, 415-419. doi: 10.2224/sbp.1998.26.4.415

Attard-Johnson, J., Bindemann, M., \& Ó Ciardha, C. (2016). Pupillary response as an age-specific measure of sexual interest: An exploratory study. Archives of Sexual Behavior.

Bailey, J. M. (2009). What is sexual orientation and do women have one? In D. A. Hope (Ed.), Contemporary perspectives on lesbian, gay, and bisexual identities (Vol. 54, pp. 43-63). New York, NY: Springer.

Bailey, J. M., Rieger, G., \& Rosenthal, A. (2011). Still in search of bisexual sexual arousal: Comment on Cerny and Janssen (2011). Archives of Sexual Behavior, 40, 1293-1295. doi: 10.1007/s10508-011-9778-5

Bradley, M. M., Miccoli, L., Escrig, M. A., \& Lang, P. J. (2008). The pupil as a measure of emotional arousal and autonomic activation. Psychophysiology, 45, 602-607. doi: $10.1111 / j .1469-8986.2008 .00654 . x$

Chivers, M. L., Rieger, G., Latty, E. M., \& Bailey, J. M. (2004). A sex difference in the specificity of sexual arousal. Psychological Science, 15, 736-744. doi: 10.1111/j.0956-7976.2004.00750.x

Chivers, M. L., Seto, M. C., \& Blanchard, R. (2007). Gender and sexual orientation differences in sexual response to sexual activities versus gender of actors in sexual films. Journal of Personality and Social Psychology, 93, 1108-1121. doi: 10.1037/0022-3514.93.6.1108 
Chivers, M. L., Seto, M. C., Lalumière, M. L., Laan, E., \& Grimbos, T. (2010). Agreement of self-reported and genital measures of sexual arousal in men and women: A meta-analysis. Archives of Sexual Behavior, 39, 5-56. doi: $10.1007 / \mathrm{s} 10508-009-9556-9$

Ebsworth, M., \& Lalumière, M. L. (2012). Viewing time as a measure of bisexual sexual interest. Archives of Sexual Behavior, 41, 161-172. doi: 10.1007/s10508-0129923-9

Friedman, M. S., Silvestre, A. J., Gold, M. A., Markovic, N., Savin-Williams, R. C., Huggins, J., et al. (2004). Adolescents define sexual orientation and suggest ways to measure it. Journal of Adolescence, 27, 303-317. doi:

10.1016/j.adolescence.2004.03.006

Goldinger, S. D., \& Papesh, M. H. (2012). Pupil dilation reflects the creation and retrieval of memories. Current Directions in Psychological Science, 21, 90-95. doi: $10.1177 / 0963721412436811$

Harris, G. T., Rice, M. E., Quinsey, V. L., Chaplin, T. C., \& Earls, C. (1992). Maximizing the discriminant validity of phallometric assessment data. Psychological Assessment, 4, 502-511. doi: 10.1037/1040-3590.4.4.502

Heaver, B., \& Hutton, S. B. (2011). Keeping an eye on the truth? Pupil size changes associated with recognition memory. Memory, 19, 398-405. doi: $10.1080 / 09658211.2011 .575788$

Henrich, J., Heine, S. J., \& Norenzayan, A. (2010). The weirdest people in the world? Behavioral and Brain Sciences, 33, 61-83. doi: 10.1017/S0140525X0999152X 
Herek, G. (2004). Beyond "Homophobia": Thinking about sexual prejudice and stigma in the twenty-first century. Sexuality Research \& Social Policy, 1, 6-24. doi: 10.1525/srsp.2004.1.2.6

Hess, E. H., \& Polt, J. M. (1960). Pupil size as related to interest value of visual stimuli. Science, 132, 349-350. doi: 10.2307/1706082

Hess, E. H., Seltzer, A. L., \& Shlien, J. M. (1965). Pupil response of hetero- and homosexual males to pictures of men and women: A pilot study. Journal of Abnormal Psychology, 70, 165-168. doi: 10.1037/h0021978

Huberman, J. S., \& Chivers, M. L. (2015). Examining gender-specificity of sexual response with concurrent thermography and plethysmography. Psychophysiology, 52, 1382-1395. doi: 10.1111/psyp.12466

Janssen, E., Prause, N., \& Geer, J. H. (2007). The sexual response. In J. T. Cacioppo, L. G. Tassinary \& G. G. Berntson (Eds.), Handbook of psychophysiology (3rd ed.). (pp. 245-266). New York, NY: Cambridge University Press.

Johnson, M. H. (1995). The inhibition of automatic saccades in early infancy. Developmental Psychobiology, 28, 281-291. doi: 10.1002/dev.420280504

Kinsey, A. C., Pomeroy, W. B., \& Martin, C. E. (1948). Sexual behavior in the human male. Philadelphia, PA: Saunders.

Korchmaros, J. D., Powell, C., \& Stevens, S. (2013). Chasing sexual orientation: A comparison of commonly used single-indicator measures of sexual orientation. Journal of Homosexuality, 60, 596-614. doi: 10.1080/00918369.2013.760324

Laeng, B., Sirois, S., \& Gredebäck, G. (2012). Pupillometry. Perspectives on Psychological Science, 7, 18-27. doi: 10.1177/1745691611427305 
Lang, P. J., \& Bradley, M. M. (2010). Emotion and the motivational brain. Biological Psychology, 84, 437-450. doi: 10.1016/j.biopsycho.2009.10.007

Lippa, R. A. (2012). Effects of sex and sexual orientation on self-reported attraction and viewing times to images of men and women: Testing for category specificity. Archives of Sexual Behavior, 41, 149-160. doi: 10.1007/s10508-011-9898-y

Lykins, A., Meana, M., \& Kambe, G. (2006). Detection of Differential Viewing Patterns to Erotic and Non-Erotic Stimuli Using Eye-Tracking Methodology. Archives of Sexual Behavior, 35, 569-575. doi: 10.1007/s10508-006-9065-z

Lykins, A. D., Meana, M., \& Strauss, G. P. (2008). Sex differences in visual attention to erotic and non-erotic stimuli. Archives of Sexual Behavior, 37, 219-228. doi: 10.1007/s10508-007-9208-X

McConaghy, N. (1999). Unresolved issues in scientific sexology. Archives of Sexual Behavior, 28, 285-318. doi: 10.1023/A:1018744628161

Mirandé, A. (2015). Hombres Mujeres: An indigenous third gender. Men and Masculinities. doi: 10.1177/1097184X15602746

Mishra, S., Clark, A., \& Daly, M. (2007). One woman's behavior affects the attractiveness of others. Evolution and Human Behavior, 28, 145-149. doi: 10.1016/j.evolhumbehav.2006.11.001

Munoz, D. P., \& Everling, S. (2004). Look away: the anti-saccade task and the voluntary control of eye movement. Nature Review Neuroscience, 5, 218-228. doi: $10.1038 / \mathrm{nrn} 1345$

Nanda, S. (1986). The Hijras of India. Journal of Homosexuality, 11, 35-54. doi: 10.1300/J082v11n03_03 
Petterson, L. J., Dixson, B. J., Little, A. C., \& Vasey, P. L. (2015). Viewing time measures of sexual orientation in Samoan cisgender men who engage in sexual interactions with Fa'afafine. PLoS ONE, 10, e0116529. doi:

10.1371/journal.pone.0116529

Petterson, L. J., Dixson, B. J., Little, A. C., \& Vasey, P. L. (2016). Reconsidering male bisexuality: Sexual activity role and sexual attraction in Samoan men who engage in sexual interactions with Fa'afafine. Journal of Sexual Orientation and Gender Diversity

Ragins, B. R., Singh, R., \& Cornwell, J. M. (2007). Making the invisible visible: Fear and disclosure of sexual orientation at work. Journal of Applied Psychology, 92, 11031118. doi: 10.1037/0021-9010.92.4.1103

Rahman, Q., \& Wilson, G. D. (2003). Born gay? The psychobiology of human sexual orientation. Personality and Individual Differences, 34, 1337-1382. doi: $10.1016 / \mathrm{S} 0191-8869(02) 00140-\mathrm{X}$

Rieger, G., Cash, B. M., Merrill, S. M., Jones-Rounds, J., Dharmavaram, S. M., \& SavinWilliams, R. C. (2015a). Sexual arousal: The correspondence of eyes and genitals. Biological Psychology, 104, 56-64. doi: 10.1016/j.biopsycho.2014.11.009

Rieger, G., Gygax, L., Linsenmeier, J., Siler-Knogl, A., Moskowitz, D., \& Bailey, J. M. (2011). Sex typicality and attractiveness in childhood and adulthood: Assessing their relationships from videos. Archives of Sexual Behavior, 40, 143-154. doi: $10.1007 / \mathrm{s} 10508-009-9512-8$ 
Rieger, G., Rosenthal, A. M., Cash, B. M., Linsenmeier, J. A. W., Bailey, J. M., \& SavinWilliams, R. C. (2013). Male bisexual arousal: A matter of curiosity? Biological Psychology, 94, 479-489. doi: 10.1016/j.biopsycho.2013.09.007

Rieger, G., \& Savin-Williams, R. C. (2012). The eyes have it: Sex and sexual orientation differences in pupil dilation patterns. PLoS ONE, 7, e40256. doi:

10.1371/journal.pone.0040256

Rieger, G., Savin-Williams, R. C., Chivers, M. L., \& Bailey, J. M. (2015b). Sexual arousal and masculinity-femininity of women. Journal of Personality and Social Psychology. doi: 10.1037/pspp0000077

Rosenthal, A. M., Sylva, D., Safron, A., \& Bailey, J. M. (2012). The male bisexuality debate revisited: Some bisexual men have bisexual arousal patterns. Archives of Sexual Behavior, 41, 135-147. doi: 10.1007/s10508-011-9881-7

Savin-Williams, R., Rieger, G., \& Rosenthal, A. M. (2013). Physiological evidence for a mostly heterosexual orientation among men. Archives of Sexual Behavior, 42, 697-699. doi: 10.1007/s10508-013-0093-1

Savin-Williams, R. C. (2016). Gay Teens: Boys to Men. Cambridge, MA: Harvard University Press.

Sell, R. L. (1997). Defining and measuring sexual orientation: A review. Archives of Sexual Behavior, 26, 643-658. doi: 10.1023/A:1024528427013

Seto, M. C., Lalumière, M. L., \& Blanchard, R. (2000). The discriminative validity of a phallometric test for pedophilic interests among adolescent sex offenders against children. Psychological Assessment, 12, 319-327. doi: 10.1037/10403590.12.3.319 
Strassberg, D., \& Lowe, K. (1995). Volunteer bias in sexuality research. Archives of Sexual Behavior, 24, 369-382. doi: 10.1007/bf01541853

Suschinsky, K. D., \& Lalumière, M. L. (2011). Prepared for anything? An investigation of female genital arousal in response to rape cues. Psychological Science, 22, 159165. doi: $10.1177 / 0956797610394660$

ten Donkelaar, H. J., Němcová, V., Lammens, M., Overeem, S., \& Keyser, A. (2011). The Autonomic Nervous System. Clinical Neuroanatomy, doi: 10.1007/19781003-1642-19134-19133_19112.

Wolchik, S. A., Spencer, S. L., \& Lisi, I. S. (1983). Volunteer bias in research employing vaginal measures of sexual arousal. Archives of Sexual Behavior, 12, 399-408. doi: $10.1007 / \mathrm{bf} 01542883$

Young, A. (2000). Women who become men: Albanian sworn virgins. Oxford, UK: Berg Publishers. 
Pupil Dilation to Explicit and Non-Explicit Sexual Stimuli

Table 1.

Correlations between Pupil Dilation, Viewing Time, Self-Reported Attraction to Explicit Sexual Stimuli, and Self-Reported Sexual Orientation across Male $(N=165$, above Diagonal) and Female $(N=160$, below Diagonal) Participants.

\begin{tabular}{|c|c|c|c|c|c|c|c|}
\hline Measure & $\begin{array}{l}\text { Pupil Dilation } \\
\text { To Same Sex }\end{array}$ & $\begin{array}{l}\text { Pupil Dilation } \\
\text { To Other Sex }\end{array}$ & $\begin{array}{l}\text { Viewing } \\
\text { Time }^{1}\end{array}$ & $\begin{array}{c}\text { Attraction to } \\
\text { Same Sex } \\
\text { Stimuli }\end{array}$ & $\begin{array}{c}\text { Attraction to } \\
\text { Other Sex } \\
\text { Stimuli }\end{array}$ & $\begin{array}{c}\text { Sexual } \\
\text { Attraction } \\
\text { Contrast }^{1}\end{array}$ & $\begin{array}{c}\text { Sexual } \\
\text { Orientation }^{1}\end{array}$ \\
\hline Pupil Dilation To Same Sex & & $\begin{array}{c}-.82 * * * \\
{[-.87,-.76]}\end{array}$ & $\begin{array}{c}.50 * * * \\
{[.37, .60]}\end{array}$ & $\begin{array}{c}.45 * * * \\
{[.32, .56]}\end{array}$ & $\begin{array}{c}-.38 * * * \\
{[-.51,-.25]}\end{array}$ & $\begin{array}{c}.52 * * * \\
{[.39, .62]}\end{array}$ & $\begin{array}{c}.50 * * * \\
{[.38, .61]}\end{array}$ \\
\hline Pupil Dilation To Other Sex & $\begin{array}{c}-.77 * * * \\
{[-.82,-.69]}\end{array}$ & & $\begin{array}{c}-.53 * * * \\
{[-.63,-.41]}\end{array}$ & $\begin{array}{c}-.44 * * * \\
{[-.56,-.31]}\end{array}$ & $\begin{array}{c}.43 * * * \\
{[.29, .54]}\end{array}$ & $\begin{array}{c}-.55^{* * *} \\
{[-.65,-.44]}\end{array}$ & $\begin{array}{c}-.54 * * * \\
{[-.64, .43]}\end{array}$ \\
\hline Viewing Time $^{1}$ & $\begin{array}{c}.41 * * * \\
{[.27, .53]}\end{array}$ & $\begin{array}{c}-.48 * * * \\
{[-.59,-.35]}\end{array}$ & & $\begin{array}{c}.77 * * * \\
{[.69, .83]}\end{array}$ & $\begin{array}{c}-.74 * * * \\
{[-.80,-.66]}\end{array}$ & $\begin{array}{c}.93 * * * \\
{[.91, .95]}\end{array}$ & $\begin{array}{c}.91 * * * \\
{[.88, .93]}\end{array}$ \\
\hline Sexual Attraction to Same Sex & $.29 * *$ & $-.39 * * *$ & $.69 * * *$ & & $-.35^{* * *}$ & $.80 * * *$ & $.75^{* * *}$ \\
\hline Stimuli & {$[.14, .43]$} & {$[-.51,-.25]$} & {$[.59, .76]$} & & {$[-.48,-.21]$} & {$[.74, .85]$} & {$[.67, .81]$} \\
\hline Sexual Attraction to Other & $-.33 * * *$ & $.29 * *$ & $-.58 * * *$ & $-.14^{\dagger}$ & & $-.77 * * *$ & $-.76 * * *$ \\
\hline Sex Stimuli & {$[-.46,-.18]$} & {$[.14, .43]$} & {$[-.67,-.46]$} & {$[-.29, .01]$} & & {$[-.83,-.70]$} & {$[-.82,-.69]$} \\
\hline Sexual Attraction Contrast ${ }^{1}$ & $\begin{array}{c}.33 * * * \\
{[.19, .47]}\end{array}$ & $\begin{array}{c}-.42 * * * \\
{[-.54,-.29]}\end{array}$ & $\begin{array}{c}.89 * * * \\
{[.85, .92]}\end{array}$ & $\begin{array}{c}.76^{* * *} \\
{[.69, .82]}\end{array}$ & $\begin{array}{c}-.61 * * * \\
{[-.70,-.50]}\end{array}$ & & $\begin{array}{c}.95 * * * \\
{[.93, .96]}\end{array}$ \\
\hline Sexual Orientation ${ }^{1}$ & $\begin{array}{c}.39 * * * \\
{[.25, .52]}\end{array}$ & $\begin{array}{c}-.44 * * * \\
{[-.56,-.31]}\end{array}$ & $\begin{array}{c}.82 * * * \\
{[.76, .87]}\end{array}$ & $\begin{array}{c}.67 * * * \\
{[.57, .75]}\end{array}$ & $\begin{array}{c}-.60 * * * \\
{[-.69,-.50]}\end{array}$ & $\begin{array}{c}.90 * * * \\
{[.86, .92]}\end{array}$ & \\
\hline
\end{tabular}

Note. Numbers in brackets represent $95 \%$ confidence intervals. ${ }^{1}$ Higher scores indicate stronger response or orientation to the same sex and less to the other sex. ${ }^{\dagger} p<.10 .{ }^{* *} p<.001 .{ }^{* * *} p<.0001$. 
Table 2.

Correlations between Pupil Dilation, Viewing Time, Self-Reported Attraction to Non-Explicit Sexual Stimuli, and Self-Reported Sexual Orientation across Male $(N=165$, above Diagonal) and Female $(N=160$, below Diagonal $)$ Participants.

\begin{tabular}{|c|c|c|c|c|c|c|c|}
\hline Measure & $\begin{array}{l}\text { Pupil Dilation } \\
\text { To Same Sex }\end{array}$ & $\begin{array}{l}\text { Pupil Dilation } \\
\text { To Other Sex }\end{array}$ & $\begin{array}{l}\text { Viewing } \\
\text { Time }^{1}\end{array}$ & $\begin{array}{c}\text { Attraction to } \\
\text { Same Sex } \\
\text { Stimuli }\end{array}$ & $\begin{array}{c}\text { Attraction to } \\
\text { Other Sex } \\
\text { Stimuli }\end{array}$ & $\begin{array}{c}\text { Sexual } \\
\text { Attraction } \\
\text { Contrast }^{1}\end{array}$ & $\begin{array}{c}\text { Sexual } \\
\text { Orientation }^{1}\end{array}$ \\
\hline Pupil Dilation To Same Sex & & $\begin{array}{c}-.20 * \\
{[-.35,-.05]}\end{array}$ & $\begin{array}{c}.22 * \\
{[.07, .36]}\end{array}$ & $\begin{array}{c}.28 * * \\
{[.13, .41]}\end{array}$ & $\begin{array}{c}-.25^{* *} \\
{[-.38,-.10]}\end{array}$ & $\begin{array}{c}.31 * * * \\
{[.16, .44]}\end{array}$ & $\begin{array}{c}.28^{* *} \\
{[.13, .41]}\end{array}$ \\
\hline Pupil Dilation To Other Sex & $\begin{array}{c}-.14^{\dagger} \\
{[-.28, .02]}\end{array}$ & & $\begin{array}{c}-.37 * * * \\
{[-.50,-.23]}\end{array}$ & $\begin{array}{c}-.29 * * \\
{[-.42,-.14]}\end{array}$ & $\begin{array}{c}.31 * * * \\
{[.16, .44]}\end{array}$ & $\begin{array}{c}-.38^{* * *} \\
{[-.51,-.24]}\end{array}$ & $\begin{array}{c}-.41 * * * \\
{[-.53,-.28]}\end{array}$ \\
\hline Viewing Time $^{1}$ & $\begin{array}{c}.29 * * \\
{[.14, .43]}\end{array}$ & $\begin{array}{c}-.22 * \\
{[-.37,-.07]}\end{array}$ & & $\begin{array}{c}.56^{* * *} \\
{[.44, .66]}\end{array}$ & $\begin{array}{c}-.62 * * * \\
{[-.71,-.52]}\end{array}$ & $\begin{array}{c}.81^{* * *} \\
{[.75, .86]}\end{array}$ & $\begin{array}{c}.77 * * * \\
{[.70, .83]}\end{array}$ \\
\hline Sexual Attraction to Same Sex & $.25^{*}$ & -.09 & $.62 * * *$ & & $-.14^{\dagger}$ & $.70 * * *$ & $.67 * * *$ \\
\hline Stimuli & {$[.10, .39]$} & {$[-.24, .07]$} & {$[.51, .71]$} & & {$[-.29, .01]$} & {$[.61, .77]$} & {$[.58, .75]$} \\
\hline Sexual Attraction to Other & $-.24 *$ & .12 & $-.44 * * *$ & $-.13^{\dagger}$ & & $-.71 * * *$ & $-.69 * * *$ \\
\hline Sex Stimuli & {$[-.38,-.09]$} & {$[-.03, .27]$} & {$[-.56,-.31]$} & {$[-.28, .02]$} & & {$[-.78,-.62]$} & {$[-.77,-.61]$} \\
\hline Sexual Attraction Contrast ${ }^{1}$ & $\begin{array}{c}.34 * * * \\
{[.19, .47]}\end{array}$ & $\begin{array}{c}-.18^{*} \\
{[-.33,-.03]}\end{array}$ & $\begin{array}{c}.78 * * * \\
{[.71, .83]}\end{array}$ & $\begin{array}{c}.78 * * * \\
{[.71, .84]}\end{array}$ & $\begin{array}{c}-.54 * * * \\
{[-.64,-.42]}\end{array}$ & & $\begin{array}{c}.92 * * * \\
{[.89, .94]}\end{array}$ \\
\hline Sexual Orientation ${ }^{1}$ & $\begin{array}{c}.36 * * * \\
{[.22, .49]}\end{array}$ & $\begin{array}{c}-.24 * \\
{[-.39,-.09]}\end{array}$ & $\begin{array}{c}.69 * * * \\
{[.60, .77]}\end{array}$ & $\begin{array}{c}.67 * * * \\
{[.58, .75]}\end{array}$ & $\begin{array}{c}-.59 * * * \\
{[-.68,-.48]}\end{array}$ & $\begin{array}{c}.87 * * * \\
{[.83, .91]}\end{array}$ & \\
\hline
\end{tabular}

Note. Numbers in brackets represent $95 \%$ confidence intervals. ${ }^{1}$ Higher scores indicate stronger response or orientation to the same sex and less to the other sex. ${ }^{\dagger} p<.10 .{ }^{*} p<.05 .{ }^{*} p<<.001 .{ }^{* * *} p<.0001$. 

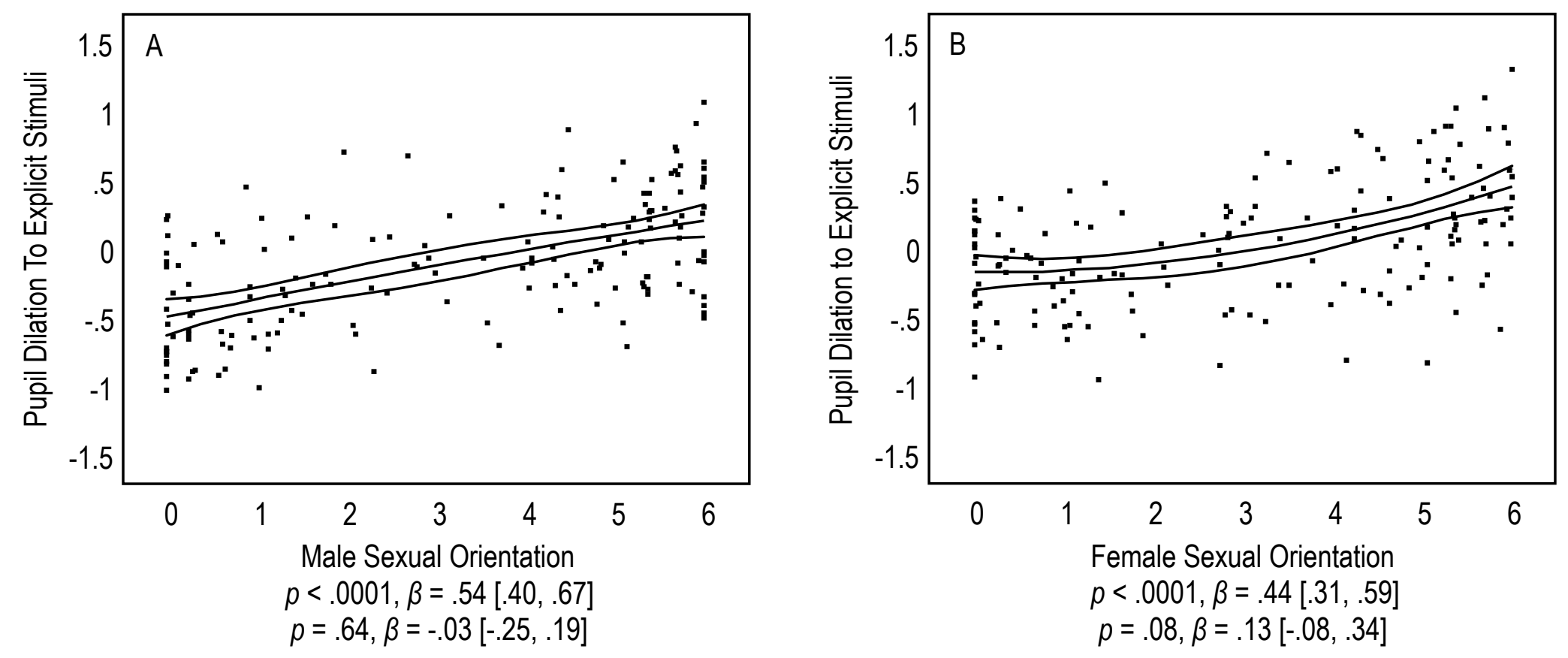

Figure 1. Reported sexual orientation of 165 men (A) and 160 women (B) relates to pupil dilation to explicit sexual stimuli of the same sex or the other sex. On the Y axes, positive numbers reflect stronger responses to the same sex, and negative numbers stronger responses to the other sex, $z$-scores within participants. On the $\mathrm{X}$ axes, 0 represents an exclusive heterosexual orientation, 3 a bisexual orientation, and 6 an exclusive homosexual orientation. Lines represent regression coefficients with 95\% confidence intervals. Dots represent participants' average scores. Statistics represent linear and curvilinear effects. 

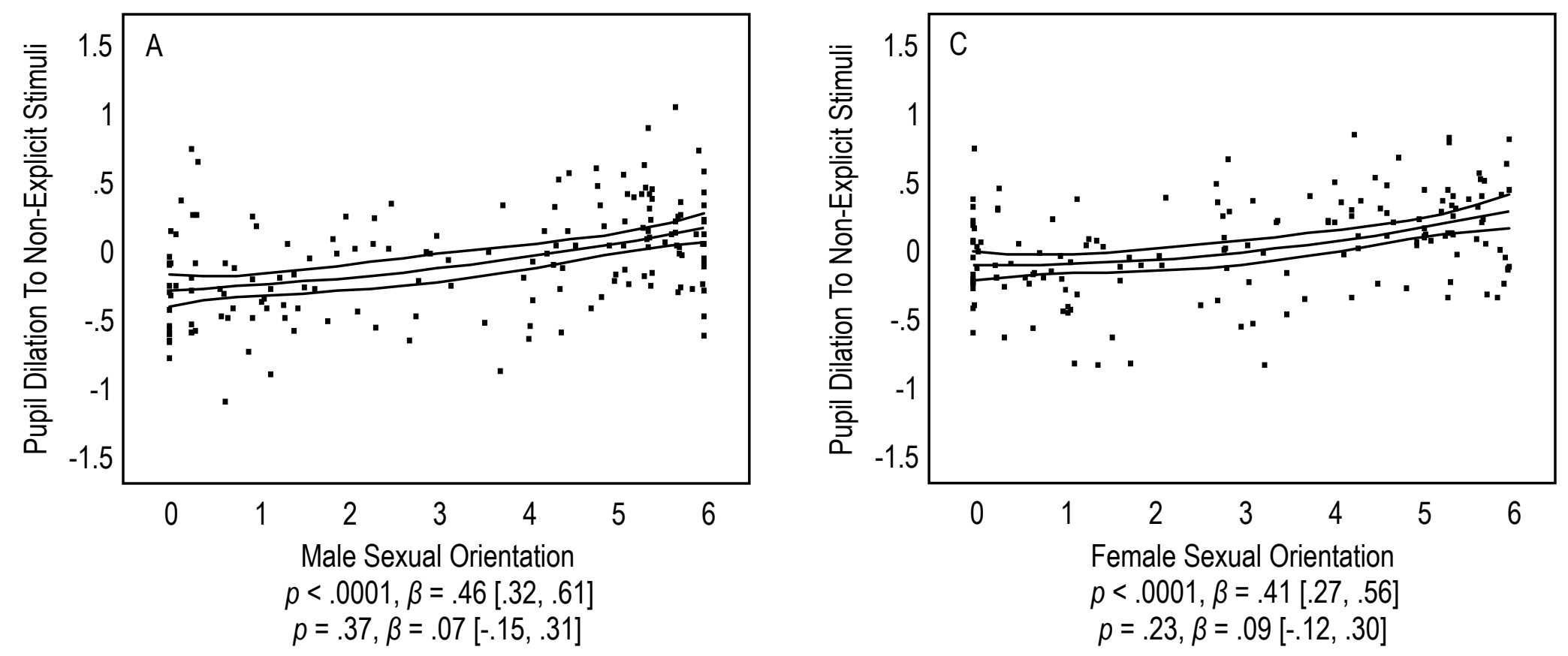

Figure 2. Reported sexual orientation of 165 men (A) and 160 women (B) relates to pupil dilation to non-explicit sexual stimuli of the same sex or the other sex. On the Y axes, positive numbers reflect stronger responses to the same sex, and negative numbers stronger responses to the other sex, $z$-scores within participants. On the $\mathrm{X}$ axes, 0 represents an exclusive heterosexual orientation, 3 a bisexual orientation, and 6 an exclusive homosexual orientation. Lines represent regression coefficients with 95\% confidence intervals. Dots represent participants' average scores. Statistics represent linear and curvilinear effects. 